\title{
The association of pathergy reaction and active clinical presentations of Behçet's disease
}

\author{
Shirin Assar ${ }^{1}$, Bahar Sadeghi' ${ }^{2}$, Fereydoun Davatchi ${ }^{2}$, Seyyedeh Zahra Ghodsi ${ }^{2}$, Abdolhadi Nadji ${ }^{2}$, \\ Farhad Shahram², Farimah Ashofte ${ }^{2}$, Seyyedeh Roghieh Larimi ${ }^{2}$, Masoud Sadeghi ${ }^{3}$ \\ ${ }^{1}$ Clinical Research Development Center, Imam Reza Hospital, Kermanshah University of Medical Sciences, Iran \\ ${ }^{2}$ Rheumatology Research Center, Tehran University of Medical Sciences, Iran \\ ${ }^{3}$ Medical Biology Research Center, Kermanshah University of Medical Sciences, Iran
}

\begin{abstract}
Objectives: The pathergy skin test is a hypersensitivity reaction to a prick skin trauma caused by a pin or a needle, which is considered as a specific presentation in Behçet's disease (BD) and the precise mechanism of this test is not well elucidated. This study was designed to evaluate the association of pathergy reaction (PR) with the active clinical manifestations of BD patients, to assess the clinical importance of PR.

Materials and methods: This was a cohort study on 1675 BD patients who fulfilled the entry criteria based on the International Criteria for Behçet's Disease (ICBD) from 1975 to 2011. The patients were divided into two groups; the pathergy positive group included 841 patients (50.2\%) and the pathergy negative group 834 patients (49.8\%). The active mucocutaneous and systemic disease manifestations were analyzed according to the presence of the PR. The odds ratio and $95 \%$ confidence interval $(95 \% \mathrm{Cl})$ were calculated for each item.

Results: In this study, 841 patients (50.2\%) had a positive pathergy test. Patient's mean age and mean disease duration were similar in the two groups (pathergy positive and pathergy negative patients). There was no association between positive pathergy test and age of patients, or disease duration. The PR was associated with male gender $(p=0.013)$, oral aphthosis $(p<0.001)$, pseudofolliculitis $(p<0.001)$, anterior uveitis $(p=0.001)$ and posterior uveitis $(p=0.028)$.

Conclusions: The presence of PR was associated with male gender, as well as some of mucocutaneous manifestations and uveitis in adult patients. There was no association with retinitis and vascular involvements. PR isn't associated with the severity of the disease.
\end{abstract}

Key words: Behçet's disease, pathergy reaction, uveitis, aphthosis.

\section{Introduction}

The pathergy skin test is a hypersensitivity reaction to a prick skin trauma caused by a pin or a needle, which is considered as a specific presentation in Behçet's disease (BD) [1]. The prevalence of a positive pathergy test (PPT) is high in the Middle East and Asian countries [2]. The precise mechanism of pathergy test (PT) is not well elucidated. A pin prick trauma in skin arouses an inflammatory response which is more pronounced in Behçet's patients than in healthy individuals. The enhanced or disproportionate release of cytokines from keratinocytes or other skin cells in epidermis or dermis can be the cause of the reaction which is observed as perivascular infiltration in skin biopsy samples [3]. Similar to other presentations of Behçet's disease, PPT is not present throughout the whole duration of the disease and is seen as a relapsing-remitting pattern [4]. The prevalence of PPT was $57.4 \%$ in Iranian, $44 \%$ in Japanese, $40 \%$ in Korean, $56 \%$ of Turkish, $68 \%$ in Moroccan, and $32 \%$ in 
the British patients [5]. The incidence of PPT was higher in the past and its presentation has shown a prominent decline over the past two decades [6]. PPT is used as a valuable diagnostic criterion for Behçet's disease. Although the decrease in the positivity of PT decreases its value as a diagnostic test, the increase of its specificity makes it more reliable [7].

\section{Aim of the study}

This study was designed to clarify the role of PT in a clinical setting and evaluate the relationship between a PPT and the active clinical presentations of the disease.

\section{Material and methods}

\section{Patients}

In a cohort study that was approved by the Ethics Committee of Tehran University of Medical Sciences, all files in the registry of BD patients, from 1975 to 2011, were checked and those fulfilling the inclusion and exclusion criteria were selected for this study.

Exclusion criteria: patients with the following criteria were excluded from the study. 1) not having a PT done 24 hours before, 2) lack of sufficient information of clinical manifestations at the same time with the PT, 3) inconclusive PT (positive or negative), 4) use of steroids, cytotoxic, or any other agents which might affect the immune system and give a negative response.

Inclusion criteria: fulfilling the ICBD criteria (the original version, officially presented in 2006 to the $12^{\text {th }}$ International Conference on Behçet's Disease in Portugal) [8].

A questionnaire, including demographic features (age, gender and disease duration), different clinical manifestations of BD, and the result of PT were included for each patient. Pathergy test was performed in all patients one day before their first visit. Disposable needles were used to perform the PT. After disinfecting skin with Povidone lodine 10\% (Betadine), three needle pricks were done on the skin of the forearm. Needles were inserted intradermally. One prick was with a 25 gauge needle, the second was with a 21 gauge needle, and the third was with a 25 gauge needle and the injection of 0.1cc of serum normal saline. Results were read 24 hours later, at the day of patient's first visit, by one of the Dermatol-

Table I. The comparison of demographic characteristics between groups

\begin{tabular}{|lccc|}
\hline Variables & $\begin{array}{c}\text { PPT } \\
(n=841)\end{array}$ & $\begin{array}{c}\text { NPT } \\
(n=834)\end{array}$ & $p$-value \\
\hline Male/ female ratio & 1.27 & 1.002 & 0.013 \\
Age $^{\dagger}$ & $32.6 \pm 9.8$ & $32.6 \pm 9.6$ & 0.992 \\
\hline
\end{tabular}

${ }^{\top}$ Mean \pm Standard deviation (years); confidence interval at $95 \%$. PPT - positive pathergy test; NPT - negative pathergy test ogists and one of the Rheumatologists of the BD Clinic. A positive result was the formation of a papule or a pustule, on the site of the needle prick, surrounded by an erythema. Patients were divided into two groups according to the result of PT, positive pathergy test (PPT) and negative pathergy test (NPT), and all clinical manifestations of $\mathrm{BD}$ at first visit were evaluated and compared between two groups. Likewise, disease duration includes the time of beginning of clinical manifestation to diagnosis of disease, was compared in two groups.

\section{Statistical analysis}

We used the $\chi^{2}$ test for qualitative variables to compare clinical presentations in the two groups of patients. The univariate logistic regression was used to analyze the relationship between duration of disease and each clinical presentation. We also calculated odds ratio (OR) and confidence interval of $95 \%(95 \% \mathrm{Cl})$ for each item. $p<0.05$ was considered statistically significant. All statistical analyses were performed with SPSS version 17 software (SPSS Inc., Chicago, IL, USA).

\section{Results}

The pathergy positive group included 841 patients (50.2\%) and the pathergy negative group 834 patients (49.8\%) (Table I). In terms of gender distribution, 785 (46.9\%) were females and 889 (53.1\%) were males. In the pathergy negative group, due to the lack of data,one patient was excluded. Therefore, there were 417 (50\%) male patients and 416 (49\%) female patients. In the pathergy positive group, 472 (56.1\%) patients were male and 369 (43.9\%) patients were female. The difference of gender distribution in the two groups was statistically significant ( $p=$ $0.013,95 \% \mathrm{Cl}: 1.052-1.546, \mathrm{OR}=1.276)$. There was no significant difference between the two groups in demographic characteristics except for gender distribution.

The frequency of clinical manifestations in each group is shown in Table II. There were statistically significant association between PPT and oral aphthosis, pseudofolliculitis, anterior uveitis, and posterior uveitis.

In order to determine the relationship between the positive pathergy test and duration of the disease (Table III), univariate logistic analysis was used and it did not show any statistically significant relationship (OR $=0.98,95 \% \mathrm{Cl}:$ 0.971-1.002, $p=0.094)$. Univariate logistic regression analysis demonstrated that the risk for development of each clinical manifestation in the positive pathergy group of BD patients do not increase with longer duration of the disease. It is noteworthy that because of limitation of active cardiac, pulmonary, neurological, gastrointestinal and epididymo-orchitis cases in this study, their clinical manifestations were excluded from this analysis. 
Table II. Comparison of clinical manifestations of the patients

\begin{tabular}{|lccccc|}
\hline Variables & $\begin{array}{c}\text { PPT } \\
(n=841)\end{array}$ & $\begin{array}{c}\text { NPT } \\
(n=834)\end{array}$ & $p$-value & $\begin{array}{c}\text { 95\%Cl } \\
(\text { Min-Max })\end{array}$ & OR \\
\hline Oral aphthosis & $567(67.4 \%)$ & $428(51.3 \%)$ & $<0.001$ & $1.610-2.393$ & 1.963 \\
\hline Genital aphthosis & $169(20.1 \%)$ & $138(16.5 \%)$ & 0.061 & $0.989-1.626$ & 1.268 \\
\hline Skin presentations & $303(36 \%)$ & $178(21.3 \%)$ & $<0.001$ & $1.670-2.580$ & 2.076 \\
\hline Skin aphthosis & $30(3.6 \%)$ & $25(3 \%)$ & 0.513 & $0.698-2.053$ & 1.197 \\
\hline Erythema nodosum & $66(7.8 \%)$ & $46(5.5 \%)$ & 0.056 & $0.981-2.153$ & 1.459 \\
\hline Pseudofolliculitis & $253(30.1 \%)$ & $131(15.7 \%)$ & $<0.001$ & $1.821-2.919$ & 2.309 \\
\hline Ocular lesions & $279(33.2 \%)$ & $343(41.1 \%)$ & 0.001 & $0.582-0.867$ & 0.711 \\
\hline Anterior uveitis & $174(20.7 \%)$ & $233(27.9 \%)$ & 0.001 & $0.537-0.843$ & 0.673 \\
\hline Posterior uveitis & $229(27.2 \%)$ & $268(32.1 \%)$ & 0.028 & $0.640-0.975$ & 0.790 \\
\hline Retinitis & $153(18.2 \%)$ & $156(18.7 \%)$ & 0.787 & $0.755-1.237$ & 0.967 \\
\hline Articular involvement & $89(10.6 \%)$ & $86(10.3 \%)$ & 0.856 & $0.753-1.408$ & 1.029 \\
\hline Vascular involvement & $9(1.1 \%)$ & $15(1.8 \%)$ & 0.210 & $0.257-1.357$ & 0.591 \\
\hline CNS involvement & $9(1.1 \%)$ & $12(1.4 \%)$ & 0.498 & $0.311-1.768$ & 0.741 \\
\hline Pulmonary involvement & 0 & 0 & - & - & - \\
\hline Gastrointestinal involvement & $6(0.7 \%)$ & $13(1.6 \%)$ & 0.102 & $0.172-1.200$ & 0.454 \\
\hline Epididymo-orchitis & $2(0.2 \%)$ & $3(0.4 \%)$ & 0.647 & $0.110-3.962$ & 0.660 \\
\hline Cardiac involvement & 0 & 0 & - & - & - \\
\hline
\end{tabular}

PPT - positive pathergy test; NPT - negative pathergy test; $\mathrm{Cl}$ - confidence interval; OR - odds ratio

\section{Discussion}

There are conflicting results between the PR and the clinical features of $\mathrm{BD}$, and information about the relationshipbetween PR and the disease activity is limited. In this study, we checked for the first time the relationship of PR with the development of active clinical manifestations of BD. There are a few studies on the relationship between the PT and clinical manifestation of BD, focusing on clinical manifestations at all course of BD, not considering its relation to active clinical manifestations [9-12]. There are some specific and unique features in this study, which are: the large sample size; looking for active clinical manifestations, concomitant with the reading of $P R$ in $B D$ patients, and the selection of patients who do not receive any immunomodulators or cytotoxic medications at the time of pathergy procedure. Therefore, our study can demonstrate more accurately the relationship between the clinical manifestations of the disease and the PT. We found that the frequency of oral aphthosis, pseudofolliculitis, anterior uveitis, and posterior uveitis was higher in BD patients with PPT, and the differences were statistically significant.

Chang et al. [9] reported that the PT was not related to any clinical manifestations. Similarly, the results of
Table III. Risk for development of each clinical manifestation and the positive pathergy test in course of Behçet's disease with duration of the disease

\begin{tabular}{|lcc|}
\hline Variables & OR* $^{*}$ & $p$-value \\
\hline Oral aphthosis & 0.986 & 0.082 \\
\hline Ocular involvement & 0.985 & 0.064 \\
\hline Anterior uveitis & 0.985 & 0.070 \\
\hline Posterior uveitis & 0.985 & 0.072 \\
\hline Retinitis & 0.986 & 0.088 \\
\hline Skin manifestations & 0.988 & 0.129 \\
\hline Pseudofolliculitis & 0.987 & 0.112 \\
\hline Erythema nodosum & 0.987 & 0.116 \\
\hline Skin aphthosis & 0.986 & 0.093 \\
\hline Joint manifestation & 0.986 & 0.094 \\
\hline Arthralgia & 0.986 & 0.094 \\
\hline Arthritis & 0.986 & 0.093 \\
\hline Genital aphthosis & 0.988 & 0.152 \\
\hline Vascular involvement & 0.987 & 0.107 \\
\hline
\end{tabular}

${ }^{*} O R$ (odds ratio) was done with a confidence interval of $95 \%$ and standard error of 0.008 
a study conducted by Krause et al. [10] on 43 patients demonstrated no difference in clinical manifestations between the two pathergy positive and pathergy negative groups. They claimed that PT probably cannot predict more severe form of the disease. Koc et al. [11] reported that the positive pathergy was significantly more common in patients with vascular presentations than without it, and another study by Nuran et al. [12] confirmed their results with $p<0.001$. In these studies, clinical manifestation were considered during all the duration of $\mathrm{BD}$.

Although in our study the sample size was very large, there was not any relationship between the results of PT and vascular disease, perhaps because we considered active vascular signs at the time of the first visit, when PT was evaluated. In our study, PPT was significantly related to the male sex, a finding in accordance with the results of the study by Davatchi et al. [13] on 6500 BD patients. The findings are also in correlation with the results of the study conducted by Gurler et al. [14] on 2147 BD patients, Yazici et al. [15] on 159 patients and Alpsoy et al. [16] on 38 patients, all in Turkey.

In the study of Alpsoy et al. [16] was demonstrated that androgen receptor index with immune-histochemistry in the biopsy of skin, in male and female patients with BD, was significantly higher compared to normal skin samples, and they concluded that androgens could play a role in higher prevalence of PPT in men. This is contradictory to the findings of Chang et al. [9] in Korea and Krause et al. [10] in Israel, both of which demonstrated no correlation between gender and the PT. In this study, there was no statistically significant relationship between PPT and the duration of the disease and this is consistent with result of a study carried out by Krause et al. [10]. More ever, in a study conducted by Davatchi et al. [17] in a 30-year period on 6057 patients, it was demonstrated that the incidence of a PPT has decreased during 30 years in Iran. However, in a study conducted by Dilsen et al. [18] in Turkey on 152 patients in 1986, it was demonstrated that if the duration of the disease was less than 5 years, the possibility of a PPT was increased.

\section{Conclusions}

The presence of pathergy reaction was associated with male gender as well as some of mucocutaneous manifestations and uveitis in BD, in adult patients, but there was no association with retinitis and vascular involvements and probably PR dose not associated with more severe disease. We should mention a limitation of our study is the small number of patients with some severe active manifestations of the disease such as neurological, pulmonary, cardiac, and gastrointestinal involvement because we select just BD patients who re- ferred to the outpatient Clinic that might be a reason for absence of statistical differences. Therefore, we couldn't demonstrate if PPT was related to these more severe clinical manifestations of BD. This factor for selection of patients should be considered in further studies. We didn't use specific disease activity indices for BD, such as the Iranian Behçet's Disease Dynamic Activity Measure (IBDDAM), to evaluate the relationship between the PT and activity of BD, and this requires further study.

This research was supported and funded by a health services grant (Code: 14417-41-02-90) from the Research Deputy of Tehran University of Medical Sciences, Tehran, Iran.

The authors declare no conflict of interest.

\section{References}

1. Davatchi F. Davatchi F. Behçet's disease. In: Ashit S.Text book of rheumatology: Principals and Practice. Japee Brotheus; 2010: 249-268.

2. Shahram F, Davatchi F, Akbarian M. The1996 survey of Behcet's disease in Iran, study of 3153 cases. In: Behçet's Disease. Hamza M (ed.). Pub Adhoua, Tunisia 1997; 165-169.

3. Gul A, Esin S, Dilsen N, et al. Immunohistology of skin pathergy reaction in Behcet's disease. Dermatol 1995; 132: 901-907.

4. Chams-Davatchi C, Davatchi F, Shahram F, et al. Longitudinal study of the Pathergy phenomenon in Behcet's disease. In: Behçet's Disease. Hamza M (ed.). Pub Adhoua, Tunisia 1997; 356-358.

5. Davatchi F, Shahram F, Chams C, et al. Behçet's disease. Acta Medica Iranica 2005; 43: 233-242.

6. Davatchi F, Shahram F, Chams-Davatchi C, et al. Behcet's disease: from east to west. Clin Rheumatol 2010; 29: 823-833.

7. Davatchi F, Chams C, Ghodsi Z. Diagnostic value of Pathergy Test in Behcet's Disease according to the change of incidence over the time. Clin Rheumatol 2011; 30: 1151-1155.

8. International Team for the Revision of the International Criteria for Behcet's Disease. Revision of the international criteria for Behcet's disease (ICBD). Clin Exp Rheumatol 2006; 24: S14-15.

9. Chang HK, Cheon KS. The Clinical Significance of a Pathergy Reaction in Patients with Behcet's Diseas. J Korean Med Sci 2002; 17: 371-374.

10. Krause I, Molad Y, Mitrani M, et al. Pathergy reaction in Behcet's disease: lack of correlation with mucocutaneous manifestations and systemic disease expression. Clin Exp Rheumatol 2000; 18: 71-74.

11. Koc Y, Güllü I, Akpek G, et al. Vascular involvement in Behçet's disease. J Rheumatol 1992; 19: 402-410.

12. Nuran A, Gunes G, Basak Y,et al. Patient characteristics in Behcet's disease. A retrospective analysis of 213 Turkish patients during 2001-4. Am J Clin Dermatol 2009; 10: 411-418.

13. Davatchi F, Shahram F, Chams-Davatchi C, et al. Behcet's disease in Iran: analysis of 6500 cases. Int J Rheum Dis 2010; 13: 367-373. 
14. Gurler A, Boyvat A, Tursen U. Clinical manifestations of Behçet's disease: an analysis of 2147 patients. Yonsei Med 1997; 38: 423-427.

15. Yazici $H$, Tuzun $Y$, Tanman AB, et al. Male patients with Behçet's syndrome have stronger pathergy reactions. Clin Exp Rheumatol 1985; 3: 137-141.

16. Alpsoy E, Elpek O, Yilmaz F, et al. Androgen receptor levels of oral and genital ulcers and skin pathergy test in patients with Behçet's disease. Dermatology 2005; 210: 31-35.
17. Davatchi F, Chams C, Shahram F, et al. Pathergy test in Behcet's disease: change in incidence over the time. APLAR J Rheumatology 2007; 10: 333-335.

18. Dilsen N, Konice M, Aral O, et al. Standardization and evaluation of the skin pathergy test in Behcet's disease and controls. In: Recent advances in Behçet's disease. Lehner T, Barnes C (eds.). Royal Society of Medicine Services, London 1986: 177180. 